\title{
FORTALECER LA COMPRENSIÓN LECTORA: UN RETO DEL MAESTRO
}

\author{
Eugenia Bermúdez Díaz
}




\section{TO STRENGTHEN READING COMPREHENSION: A CHALLENGE FOR THE TEACHER}

\section{ABSTRACT}

This article describes the impact of the teaching sequence as a strategy used by the author, to strengthen reading comprehension processes in the moments before, during and after reading, in the third grade students of the school Gonzalo Jimenez Navas de Floridablanca. Through their experience you seek to raise awareness of the importance of the use of motivational strategies in the classroom to develop the pleasure of reading and thus strengthen it.

Key words: : strategies, teaching sequence, process, reading comprehension.
RESUMEN

Este artículo describe la incidencia de la secuencia didáctica como estrategia utilizada por la autora para fortalecer los procesos de comprensión lectora en los momentos antes, durante y después de la lectura, en los estudiantes de tercer grado del colegio Gonzalo Jiménez Navas de Floridablanca. A través de su experiencia busca crear conciencia de la importancia que tiene el uso de estrategias motivadoras en el aula para desarrollar el placer por la lectura y por ende fortalecerla.

Palabras clave: estrategias, secuencia didáctica, procesos, comprensión de lectura.

\section{AUTOR}

\section{Eugenia Bermúdez Díaz}

Especialista en Administración de la Tecnología Educativa. Universidad de Santander. Docente Colegio Gonzalo Jiménez Navas. Floridablanca Santander.

Correo electrónico: aebermudez309@unab.edu.co 


\section{INTRODUCCION}

Una de las dificultades que experimentan niños y jóvenes es la comprensión de textos, ellos manifiestan desinterés por la lectura, la consideran como un proceso que ya lo saben hacer, por el simple hecho de identificar los signos gráficos por un nombre o por un sonido y que no necesitan practicarla para mejorarla; de ahí, las grandes deficiencias tanto en velocidad como en comprensión, es entonces, cuando la escuela empieza a jugar un papel importante para desarrollar esta habilidad, debido a que los hábitos lectores en los niños no se desarrollan de la noche a la mañana, son un proceso que debe iniciar en el hogar y continuar en el colegio, donde se fortalecen cuando se usan las estrategias adecuadas que le permiten a los niños ver la lectura como una acción significante, portanto, no es una cuestión mecánica ni de mera habilidad; es un proceso que permite desarrollar habilidades y destrezas para el análisis, la comprensión y la adquisición de conocimientos; y no como un castigo.

En este sentido el maestro deberá buscar estrategias no sólo para continuar desarrollando hábitos lectores sino para hacer de la lectura en primera instancia momentos de placer, orientando al estudiante para que descubra por sí mismo que leer sirve para algo, que aporta información que luego se transformará en conocimiento, es decir, que el estudiante descubra la utilidad de la lectura y el uso que le puede dar, de ahí que fortalecer los procesos de comprensión lectora son fundamentales desde los primeros años de edad escolar, puesto que éstos inciden de manera directa en diferentes aspectos del aprendizaje como lo es, el rendimiento académico, la atención y la memoria. Al respecto Goodman (1996) opina que "los niños que aprenden a disfrutar la lectura, llegado el momento de elegir, elegirán leer. Aquellos que aprenden a leer, pero no a disfrutar de la lectura, rara vez elegirán leer durante su tiempo libre" (p. 46). Es por esto que mejorar los procesos de comprensión lectora se ha convertido en un desafío no solo para las instituciones educativas sino también para el gobierno, ya que a través de proyectos como el Plan Nacional de Lectura y Escritura (PNLE) se pretende mejorar las competencias comunicativas de niños y jóvenes, buscando desempeños altos en las diferentes pruebas nacionales e internacionales a las que ellos deben enfrentarse en su vida escolar y por ende mejorar la calidad de educación de nuestro país.

De acuerdo con lo anterior, es fundamental implementar en la institución una serie de estrategias que permitan en el estudiante el desarrollo y fortalecimiento de la habilidad para comprender e interpretar textos; de ahí que la propuesta de investigación tome específicamente el género narrativo, teniendo en cuenta que este género concuerda y armoniza con la capacidad de crear fantasías, capacidad que ya está presente desde la infancia, Egan (1991), señala que "la mente infantil es narrativa, competencia que le permite interactuar con facilidad con los relatos y producir narraciones orales y escritas, además es este tipo de texto muy atractivo por su estilo y el que más llama la atención; por tanto se parte del interés del niño y se hace necesario aprovecharlo para orientar su análisis hacia la comprensión lectora.

La propuesta está encaminada a fortalecer los procesos de comprensión lectora en los estudiantes de tercer grado de la institución educativa, mediante la implementación de la secuencia didáctica en los momentos antes, durante y después de la lectura, de textos narrativos a través de la experiencia en el aula. Vale la pena resaltar que los textos seleccionados para la implementación de la secuencia didáctica pertenecen a la literatura infantil, escritos por la argentina María 
Elena Walsh; se eligió esta autora puesto que su estilo narrativo atiende los elementos ideológicos contextuales que permite sumergirse en el mágico mundo de la fantasía, el descubrimiento y la imaginación.

\section{METODOLOGÍA}

Este trabajo de investigación estuvo enmarcado desde la Investigación Acción de tipo cualitativo. La finalidad de este tipo de investigación es recoger, revisar, analizar e interpretar los resultados que caracterizan un hecho, fenómeno o grupo, con el fin de conocer su comportamiento y aportar una solución al problema encontrado, para lo cual se hizo necesario tener en cuenta los planteamientos que hacen algunos teóricos sobre la Investigación Acción (I-A):

\begin{abstract}
Elliott, el principal representante de la investigaciónacción desde un enfoque interpretativo define la investigación-acción como "un estudio de una situación social con el fin de mejorar la calidad de la acción dentro de la misma". La entiende como una reflexión sobre las acciones humanas y las situaciones sociales vividas por el profesorado que tiene como objetivo ampliar la comprensión (diagnóstico) de los docentes de sus problemas prácticos. Las acciones van encaminadas a modificar la situación una vez que se logre una comprensión más profunda de los problemas. (Murillo, et., al, 20102011)
\end{abstract}

En otras palabras, las actividades que se planean en la institución educativa durante el proceso de la I.A. tienen en común, la identificación de una situación problemática, la cual requiere de la implementación de unas estrategias de acción en el aula para ser sometida a la observación, autorreflexión y cambio, con el fin de beneficiar a una determinada población.
De ahí, que desarrollar los hábitos lectores en los niños y fortalecer los procesos de comprensión de lectura se ha convertido en un verdadero reto para los maestros. Por ello la lectura es una de las preocupaciones que todos los días está presente en las aulas de clase: ¿por qué no les gusta leer a los niños?, ¿por qué tienen dificultades para comprender un texto?, ¿cómo planear una clase donde leer tenga sentido?; cuando un niño manifiesta que "no le gusta leer" se deben considerar varias razones, entre las cuales el papel del maestro como mediador cobra gran valor, al igual que la cultura de lectura de sus padres. De ahí que una de las tareas más importantes que tiene la escuela es formar lectores.

\section{Lomas (1999) expresa que:}

No debemos olvidar que la escuela se convierte para muchos niños y jóvenes en el único escenario en el que ellos van a tener la posibilidad de acercarse al conocimiento y la lectura de textos literarios así como a la experiencia de la creación literaria, entonces es una responsabilidad grande, como maestros, hacer que esos niños y jóvenes gocen en la escuela del placer de leer y de escribir con sentido, así como es una necesidad urgente que nosotros los maestros entendamos que los tiempos han cambiado y que hay nuevas formas de aprender y de enseñar para hacer posible una sociedad que pueda participar de la verdadera ciudadanía (citado en MEN, 2013).

En este sentido se hace necesario aclarar conceptos como los de lectura, comprensión de lectura y estrategias adecuadas para lograr nuestro objetivo: fortalecer los procesos de comprensión lectora. Pero para cumplir este objetivo es necesario generar en el niño hábitos de lectura, los cuales beneficia no solo al estudiante sino también al maestro. Poder generar este hábito requiere de un proceso continuo, voluntario, no impuesto, con etapas claramente definidas que 
permitan ir avanzando e incrementando el interés del niño por la lectura. Para llegar a un feliz término de este proceso continuo, se hace necesario seleccionar textos acordes a la edad y a los intereses particulares del niño.

El hábito de la lectura como los demás buenos hábitos se desarrollan de manera espontánea, en un ambiente de total armonía, confianza y motivación permanente. Esto implica que la metodología aplicada sea flexible y acorde con los avances y logros del niño. Para fortalecer este hábito, se implementó durante el proceso de investigación una estrategia denominada "la caja viajera", que consistió en realizar la lectura diaria en familia de un texto narrativo, previamente seleccionado por la docente investigadora, el cual debía ser narrado por el estudiante al día siguiente. Durante el tiempo en que se implementó la estrategia, no solo se observaron avances a nivel de oralidad, sino también a nivel de motivación hacia la lectura, evidenciándose en la actitud que mostró gran parte de los estudiantes objeto de estudio, pues diariamente se dirigían de manera voluntaria a la biblioteca de la institución a pedir prestados libros para leer en su tiempo libre; de igual manera, los estudiantes se mostraron más participativos y cada vez más interesados por hacer anticipaciones a las lecturas diarias.

Es importante tener presente que para el desarrollo de este proceso de aprendizaje se hizo necesario romper la formalidad y la monotonía mediante actividades que llamaron la atención del niño. Veamos entonces,

\section{¿Qué es leer?}

Leer es una actividad de construcción de significados, es descubrir nuevos mundos, pero también es una actividad que nos permite redescubrir lo que ya sabemos. Aprender a leer y leer no es lo mismo. El maestro enseña al niño en sus primeros años de edad escolar a reconocer un sistema de representación escrita, pero no le ha enseñado verdaderamente a leer si esta práctica no tiene propósitos claros de construcción del conocimiento. Es entonces cuando surge la pregunta:

\section{¿Qué es comprender?}

De manera equivocada y a través del tiempo, los maestros han considerado que responder preguntas, recordar y repetir una información o memorizarla es comprender; es necesario recordar para comprender, pero no es suficiente. Entonces, dar respuestas a una serie de preguntas, es distinto a construir conocimiento. De lo anterior se puede deducir que comprender es el proceso que hace el lector de construir el conocimiento a partir de su interacción con el texto para dar respuesta a sus intereses. Pero, ¿cómo lograrlo? De ahí la importancia del maestro como agente mediador para comprender la lectura y de las estrategias que éste utilice para hacer de la lectura no solo un momento de placer sino también de construcción del conocimiento.

Este es el reto, ¿está el maestro aplicando las estrategias adecuadas para la comprensión lectora?, entonces,

\section{¿Qué es una estrategia?}

Solé (2003), sostiene que las estrategias de aprendizaje son utilizadas como "procedimientos de carácter elevado que implican la presencia de objetivos que cumplir, la planificación de las acciones que se desencadenan para lograrlos, así como su evaluación y posible cambio" (p. 59). De ahí se puede afirmar que la flexibilidad y la intencionalidad son características de las estrategias ya que éstas están encaminadas al logro de un objetivo y sujetas a cambio en el caso que no cumplan con el fin o propósito de aprendizaje. 
Resulta oportuno reflexionar en torno a ¿están los maestros usando las estrategias adecuadas para enseñar a leer y a comprender?, ¿revisan con frecuencia sus prácticas?, ¿en qué están fallando? Responder a estas preguntas conlleva a encontrar un conjunto de factores asociados entre los cuales es necesario reconocer, sin temor a una equivocación que la mayoría de los maestros continúan utilizando el método tradicional para la enseñanza del aprendizaje de la lectura; es evidente que las prácticas tradicionales en la enseñanza de la lectura y el uso de métodos silábicos y de decodificación de palabras producen una lectura lenta y monótona , y representan menos esfuerzo, por consiguiente satisface a muchos padres; pues muchos de ellos, no diferencian la decodificación, de la lectura y mucho menos de la comprensión de lectura; sin embargo no hay que desconocer los esfuerzos que hacen muchos docentes al implementar prácticas innovadoras en el aula. Es por esto, que este trabajo de investigación se centró en la implementación de la secuencia didáctica como estrategia de fortalecimiento de la comprensión lectora, tomando como base los textos narrativos debido a que éstos están cargados de fantasía y les llama la atención a los niños, y teniendo presente los diferentes momentos: antes, durante y después de la lectura. Para Solé (1992),

Antes de la lectura: este subproceso busca captar en los niños la atención, despertar su interés y activar los conocimientos previos, así mismo trata de establecer los objetivos de la lectura. ¿Para qué se lee?, ¿qué se sabe del texto?, ¿de qué se trata el texto?, ¿qué dice su estructura?

Durante la lectura: en este subproceso es donde se comprueba si la información entregada a partir de la activación de conocimientos previos coincide con la que está entregando el texto, es conveniente formular hipótesis, hacer predicciones sobre el texto, plantear preguntas sobre lo leído, esto permite aclarar dudas acerca del texto. De igual manera se hace necesario identificar lo principal de lo secundario del texto.

Después de la lectura: en esta etapa el lector está en condiciones de responder a preguntas como, ¿cuál es la idea principal?, ¿cuáles son las ideas secundarias? Se trata de organizar de manera lógica la información contenida del texto leído a través de resúmenes interpretativos, se formulen interrogantes y respondan preguntas y utilicen ordenadores gráficos para dar cuenta de lo comprendido.

Sin lugar a dudas, vencer el método tradicional y arriesgarse a implementar estrategias diferentes en el aula de clase, es el reto del maestro para fortalecer los procesos de comprensión lectora. Es ahí donde el papel del maestro como mediador es determinante para que el estudiante desarrolle el proceso de comprensión.

Para la puesta en marcha de este proyecto de investigación se partió de la observación participante y se materializó con la aplicación de una prueba diagnóstica con el fin de conocer el nivel de comprensión lectora que tenían los estudiantes de tercer grado, antes de implementada la estrategia; a partir de los resultados arrojados en el diagnóstico, donde se evidenciaron dificultades de comprensión de lectura a nivel literal, inferencial y crítico intertextual, se procedió a diseñar e implementar una secuencia didáctica que consta de catorce (14) sesiones de trabajo, siete (7) de las cuales fueron puestas en práctica en el aula y siete (7) sesiones organizadas para ser puestas en acción. A continuación se hacen explícitas cada una de las sesiones y actividades que se desarrollaron durante la implementación de la secuencia didáctica. 


\section{FASE DE PRESENTACIÓN: Antes de la lectura}

Esta fase está compuesta por dos sesiones, la primera titulada: el renacer literario, cuyo objetivo es despertar el interés en los estudiantes para la realización de las actividades propuestas. Para esta sesión se invitó a un exalumno de la I.E. que por su trayectoria como escritor, poeta, declamador y cantautor fue ejemplo motivador para el desarrollo de las actividades a realizar desde su experiencia de vida, mediante un diálogo ameno que resolvió inquietudes de los estudiantes. En la segunda sesión: Conociendo al autor y sus textos se abordó a la autora María Elena Walsh y sus textos narrativos, a partir de la interacción con uno de sus cuentos. Así mismo se propuso una actividad alterna durante la implementación de la secuencia didáctica como lo es la caja viajera que consistió en llevar a casa dentro de una caja un libro de la autora, para leer un capítulo o uno de sus cuentos en familia y luego compartir lo leído al día siguiente con sus compañeros.

\section{FASE DE COMPRENSIÓN: Durante la lectura}

Durante la tercera sesión para verificar hipótesis se propuso la actividad: A leer y comprender se dijo donde se inició recordando al autor y su contexto, posteriormente se dio conocer el título del texto y a partir de él surgieron hipótesis sobre su contenido, las cuales fueron aclaradas en el momento en que se observó un video y se leyó el cuento Gatopato y la princesa Monilda (Walsh, s.f), de igual manera el estudiante se fue apropiando de herramientas necesarias para abordar el texto e interactuar con él de una manera más efectiva. Se realizaron actividades individuales y grupales de interrogación, lúdicas y de reflexión.

\section{FASE PRÁCTICA: Después de la lectura}

En las sesiones 4 y 5 mediante el juego: Alcance la estrella se pretendió extraer información del texto. Las preguntas están diseñadas no solo para extraer información literal del texto sino para que inicien un nuevo proceso de aprendizaje a nivel inferencial. De igual manera hay preguntas que se aproximan al nivel crítico intertextual cuando se interroga sobre la actitud de personajes y su posición al respecto. En este caso el estudiante sin dejar a un lado lo literal fue alcanzando el nivel inferencial y dejando entre ver su postura frente a ciertas actitudes que se presentan en el texto. Igualmente fue un momento propicio para reflexionar sobre la importancia de ganar o perder en el juego, pues, por ser niños siempre quieren ganar. En este espacio se propuso poner en juego la imaginación para crear otro final al cuento; finalmente se compartieron los finales propuestos con el grupo.

La sesión seis se desarrolló mediante la solución de un crucigrama correspondiente al texto leído, a través de un trabajo grupal. Adicionalmente se realizó una reflexión con preguntas como: ¿cómo se sintieron en el proceso?, ¿qué aprendieron?, ¿cómo lo aprendieron?, ¿qué le cambiarían?, ¿qué faltó?, ¿es importante realizar actividades antes, durante y después de la lectura para mejorar la comprensión de un texto?, ¿por qué?

\section{FASE DE TRANSFERENCIA: Después de la lectura}

Con esta fase culminó la secuencia didáctica y se abordó mediante la realización de una serie de preguntas, se sugirió crear un nuevo cuento donde la creatividad y la imaginación fueron la pieza clave para la producción textual, es importante recalcar que la orientación del maestro fue fundamental para el 
logro del objetivo; una vez realizada la actividad se compartieron las producciones las cuales fueron recopiladas por la docente para emprender un largo camino, esta vez con el libro viajero creado por los estudiantes, con el fin de ser leído en familia. Una vez finalizado el viaje por todos los hogares se le entregó una réplica del libro a cada estudiante.

Vale la pena resaltar la integración que se dio entre los momentos de la lectura planteados por Solé (1992) y las fases o etapas de la secuencia didáctica; en cada una de ellas se desarrollaron actividades encaminadas a fortalecer los procesos de comprensión de lectura. Finalizadas cada una de las sesiones se realizó una reflexión en el diario de campo, reflexión que permitió a la docente investigadora comprobar si la estrategia aplicada fue la más acertada o no, se realizaron cambios pertinentes que permitieron encontrar resultados positivos.

\section{RESULTADOS}

Finalizada la propuesta de investigación se aplicó una prueba final o post test con el objetivo de valorar el estado final de la comprensión lectora en textos narrativos, el cual evidenció:

El avance en el nivel literal, puesto que, a los estudiantes se les facilitó reconocer los elementos (personajes, características de los personajes, espacio, tiempo y lugar) y los momentos (inicio, nudo y desenlace) que componen el género narrativo.

En cuanto al nivel inferencial, los estudiantes objeto de estudio, mostraron un avance significativo con respecto al nivel inicial, ya que lograron hacer deducciones y dar cuenta de diversas situaciones expuestas en el texto. En el nivel crítico el avance fue significativo, los estudiantes lograron desarrollar la competencia propositiva y relacionar los saberes previos con los obtenidos en el texto, para producir nuevo conocimiento.

\section{CONCLUSIONES}

Una vez finalizada la propuesta de investigación se pudo concluir que:

La aplicación de la secuencia didáctica, mediante el uso del género narrativo fue una estrategia adecuada para el fortalecimiento de los procesos de comprensión lectora, puesto que, se partió de los intereses de los estudiantes, debido a que es un género que les llama la atención por las características especiales que éste posee y le permite al niño desarrollar su imaginación, fantasía y creatividad, entre otros.

Actividades como la visita del escritor y la caja viajera planteadas en la propuesta pedagógica, lograron despertar el interés y la motivación hacia la lectura.

Con la aplicación de estas estrategias se logró en el estudiante el desarrollo de los niveles de comprensión (literal, inferencial y crítico intertextual) de manera simultánea, trayendo como consecuencia mejores resultados en el post test.

Se evidenció un avance significativo en cuanto a oralidad en estudiantes con necesidades educativas especiales, lo que permite inferir que la estrategia utilizada aportó para mejorar el desempeño de estos estudiantes.

En algunos estudiantes la estrategia aplicada no funcionó debido al alto porcentaje de inasistencia a clase que presentaron durante la puesta en marcha de la secuencia didáctica. 
Las dificultades que mostraron los estudiantes antes de ser implementada la secuencia didáctica son el resultado de prácticas tradicionales para la enseñanza de la lectura por parte de los maestros.

Es importante que el maestro utilice estrategias motivadoras para incentivar el gusto y posterior hábito por la lectura, de esta manera las dificultades para la comprensión de textos disminuiría.

Teniendo en cuenta las consideraciones anteriores, se propone a las directivas de la institución educativa, implementar esta propuesta desde los primeros años de edad escolar hasta finalizar la educación media, ya que permite no solo fomentar la comprensión de textos narrativos sino que es accesible a todo tipo de texto (argumentativo, informativo, descriptivo, etc.), además favorece el aprendizaje significativo y deja a un lado la enseñanza de la lectura como actividad repetitiva y memorística que limita el crecimiento intelectual de los estudiantes al quedarse en la lectura a nivel literal sin ahondar en lo profundo del texto que es lo que permite lectores críticos y autónomos.

De esta manera se da cumplimiento a los estándares básicos de competencias propuestos por el MEN para cada grado y niveles y adicionalmente permitirá a los estudiantes obtener mejores resultados en las pruebas nacionales (SABER) e internacionales (PISA).

\section{REFERENCIAS}

Camps, A. et al. (2006). Secuencias didácticas para aprender gramática. Barcelona:Grao.

Egan, K. (1991). La comprensión de la realidad en la educación infantil y primaria. Madrid, Ministerio de Educación y Ciencia y Ediciones Morata.
Goodman, Kenneth. (1996). La lectura, la escritura y los textos escritos: una perspectiva transaccional socio psicolingüística. En Textos en contexto 2, Los procesos de lectura y escritura. Buenos Aires: IRA.

Lomas, C. (1999). En: MEN (2013). Leer para comprender. Escribir para transformar. Bogotá: Libros Maestros. Plan nacional de Lectura y Escritura.

Martínez M. (2002). Estrategias de lectura y escritura de textos. Perspectivas teorías y talleres. Cali, Colombia: Universidad del Valle. Obtenida de: http:// www.ceip.edu.uy/IFS/documentos/2015/lengua/bibliografia/martinez_estrategiaslecturaescritura.pdf

Murillo, F. et., al (2010-2011). Investigación acción. Consultado en: https://www.uam.es/personal_pdi/ stmaria/jmurillo/InvestigacionEE/Presentaciones/Curso_10/Inv_accion_trabajo.pdf

Smith, F. (1983). Comprensión de la Lectura. México: Trillas.

Solé, I. (1992). Estrategias de lectura. Barcelona. Primera edición

(2003). Estrategias de lectura. Barcelona. Grao

Walsh, M. (s.f.). "Gatopato y la princesa Monilda". En: Mi mochila digital. Consulta en línea: http://www.mimochiladigital.com.ar/mi-lugar/pdf/1-Lenguaje/Cap5. pdf

Citar este artículo como: Bermúdez, E. (2017). “Fortalecer la comprensión lectora: un reto del maestro". En: Revista La Tercera Orilla (18). Bucaramanga: Universidad Autónoma de Bucaramanga. 